\title{
LA TAREA DOCENTE Y EL CÁLCULO DIFERENCIAL VINCULADOS CON EL PERFIL DE LAS CARRERAS BIOLÓGICAS
}

\author{
Reinaldo Sampedro Ruiz \\ reinaldo.sampedro@matcom.uh.cu \\ http://orcid.org/0000-0002-5650-5249 \\ María Elvira Fernández Sa \\ http://orcid.org/0000-0001-5811-6418 \\ Marleny Soler Martínez \\ http://orcid.org/0000-0001-7467-8488 \\ Universidad de la Habana Cuba \\ Cuba
}

Recibido: 2020-03-20; Aceptado: 2020-11-12

\begin{abstract}
Resumen
La necesidad de incluir la asignatura Cálculo Diferencial en el primer año de las Licenciaturas de Biología, Microbiología y Virología y Bioquímica y Biología Molecular está justificada por la creciente importancia de la aplicación de herramientas matemáticas en la investigación de fenómenos, procesos y conceptos biológicos. Para lograr un proceso de enseñanza-aprendizaje significativo de esa asignatura se hace necesario esclarecer desde un primer momento el vínculo de los conceptos básicos tales como función, límite, derivada, y optimización con el perfil de esas carreras. Una forma efectiva de hacerlo es a través de ejercicios o tareas docentes, donde las funciones no sean entes abstractos, sino que modelen un problema físico, biológico o químico en particular. En este trabajo se deja constancia de la introducción de un conjunto de tareas docentes diseñadas con este objetivo en el proceso de enseñanza- aprendizaje de la asignatura Cálculo Diferencial, se describen estas tareas y presentan los principales resultados obtenidos de su implementación.

Palabras clave: Tarea Docente; Cálculo Diferencial; Ciencias Biológicas

\section{A TAREFA DOCENTE E O CALCULO DIFERENCIAL VINCULADOS COM O PERFIL DOS CURSOS DE BIOLOGIA}

\section{Resumo}

A necesidade de incluir a disciplina Cálculo Diferencial no primeiro ano das Licenciaturas de Biologia, Microbiologia e Virologia, e Bioquímica e Biologia Molecular tem como justificativa a importância crescente da aplicação de ferramentas matemática na pesquisa dos fenómenos, processos e conceitos biológicos. Para conseguir um processo de ensino-aprendizagem significativa de essa disciplina se faz necessário esclarecer, desde o primeiro momento, o vinculo dos conceitos básicos tais como função, limite, derivada e optimização com o perfil de esses cursos. Uma forma efetiva de fazer isso é usando exercícios ou tarefas docentes, donde a funções não sejam entidade abstratas, senão que façam a modelagem de um problema fisico, biológico ou químico em particular. Neste trabalho se deixa constância da introdução de um 
conjunto de tarefas docentes desenhadas com esse objetivo no processo de ensino-aprendizagem da disciplina Cálculo Diferencial, são descritas essas tarefas y se apresentam os principais resultados obtidos na sua implementação.

Palavra chave: Tarefa Docente. Cálculo Diferencial. Biológicas.

\title{
THE EDUCATIONAL TASK AND THE LINKED DIFFERENTIAL CALCULATION WITH THE PROFILE OF THE BIOLOGICAL CAREERS
}

\begin{abstract}
The necessity to include the subject Differential Calculation in the first year of the Degrees of Biology, Microbiology and Virology and Biochemistry and Molecular Biology is justified by the growing importance of the application of mathematical tools in the investigation of phenomena, processes and biological concepts. To achieve a process of significant teachinglearning of that subject it becomes necessary to clarify from a first moment the bond of the such basic concepts as function, limit, derived, and optimization with the profile of those careers. An effective form of making it is through exercises or educational tasks, where the functions are not abstract entities, but rather they model a physical, biological or chemical problem in particular. In this work perseverance of the introduction of a group of educational tasks is left designed with this objective, in the teaching process - learning of the subject Differential Calculation, these tasks are described and they present the main obtained results of its implementation.
\end{abstract}

Keywords: Educational Task; Differential Calculation; Biological Sciences

\section{Introducción}

En la dinámica de la formación de los profesionales es muy importante la motivación, la comprensión y la sistematización de los contenidos. En la categoría motivación del contenido, la acción del profesor es fundamental. Si lo cognitivo es lo que está relacionado con la evolución de los procesos mentales y la capacidad del individuo para aprender y solucionar problemas, está claro que mientras más motivado esté el estudiante por alcanzar ese conocimiento, con más eficiencia lo logrará. Motivarse por algo es sentir afecto por ese tema, encariñarse, desear estar en contacto con él. Si estamos en el contexto de la Licenciatura en Bioquímica y Biología Molecular, y siguiendo la línea de pensamiento de la frase anterior, la enseñanza de la Matemática no puede desligarse del perfil vocacional si queremos potenciar el proceso de enseñanza-aprendizaje (PEA).

Para los estudiantes que disfrutan, que establecen un vínculo afectivo con el profesor, la mayor satisfacción reside en la asimilación de los contenidos. El dominio de las habilidades como vía fundamental para resolver problemas se convierte en una necesidad, pues el estudiante estará convencido de que el contenido que asimila se convertirá en la herramienta fundamental para desarrollar su labor futura. 
A partir de los elementos aportados teniendo en cuenta la experiencia como docentes y los textos didácticos que abordan el tema de la enseñanza de la Matemática en este contexto se confirma la importancia de la elaboración de un conjunto de tareas docentes que:

- Vinculen el contenido de la asignatura de Matemática con el perfil del profesional.

- Tengan carácter desarrollador.

- Tengan en cuenta los intereses personales de los estudiantes para lograr no solo conocimientos y habilidades, sino también implicación personal y corriente afectiva con el estudiante.

- Estimulen el razonamiento, el pensamiento y la independencia del estudiante.

\section{Relación del concepto de límite en el infinito de una función de variable real que modela problemas biológicos}

La tarea docente en cualquier PEA es la manera que tiene el profesor de lograr que el estudiante se apropie del contenido que acaba de enseñar. Como tal, está relacionada con la etapa de apropiación del contenido en que se encuentre ese estudiante. De ahí que se prefieren tareas de tipo algorítmico o repetitivo para un primer acercamiento a ese contenido, o sea, cuando se está en la etapa Base Orientadora de la Acción (BOA), en la cual los estudiantes se adiestran en los conceptos esenciales recibidos, así como en las acciones fundamentales.

En esta Etapa Base Orientadora de la Acción pueden sugerirse ejercicios con textos, que aunque involucren una ejecución de tipo algorítmico, relacionen la resolución de esta tarea con un problema tomado de la vida real. Para ilustrar lo anterior se muestran ejemplos donde se relaciona el cálculo de límites en el infinito con situaciones de la vida real.

Ejemplo 1 (Elaborado a partir de un modelo real)

La siguiente función $f(t)=2.49 t^{0.25} e^{-0.0062 t}$ se utiliza para describir la producción de leche en kilogramos de cierta variedad de búfalas en el instante de tiempo $t$ que se mide en días. Demuestre matemáticamente que al pasar una gran cantidad de días la producción de leche de la búfala será casi nula. 
Ejemplo 2 (Elaborado a partir de un modelo real)

En un ambiente con capacidad de carga $\mathrm{K}$, el crecimiento de una población con tamaño inicial N0 y tasa de crecimiento per cápita r puede ser modelado mediante la función:

$$
N(t)=\frac{K}{1+\left(\frac{k}{N_{0}}-1\right)^{-r t}}
$$

a) Demuestre que $\mathrm{K}$ es una asíntota horizontal de $\mathrm{N}(\mathrm{t})$

b) Explique qué significa dentro del fenómeno que se estudia.

Ejemplo 3 (Elaborado a partir de un modelo real)

En cierto análisis clínico la expresión $C(t)=53.8\left(1-e^{-0.0519 t}\right)$ se utiliza para conocer la concentración de glucosa (mg x minuto x litro de sangre) en cada momento de tiempo. ¿Después de inyectarse la dosis de glucosa qué sucede al pasar un tiempo considerablemente grande?

Este conjunto de tareas docentes elaboradas por los autores a partir de modelos reales aborda los siguientes contenidos:

- Calcular límites en el infinito de funciones de una variable real.

- Identificar situaciones de la vida real donde un concepto matemático ayuda a entender un fenómeno determinado aplicado a la futura profesión del estudiante.

- Aplicar el concepto de límite en el infinito de funciones de una variable real para resolver un ejercicio con texto relacionado con las Ciencias Naturales.

Para desarrollar estos ejercicios el estudiante debe transitar por las etapas de Motivación, Orientación, Ejecución y Control. Al diseñar ejercicios en donde el cálculo de límites se realice en el contexto de una situación vinculada con las Ciencias Naturales. El éxito de lo anteriormente expresado, estará muy vinculado al hecho de que exista la motivación constante del estudiantado hacia el objetivo de la actividad, lo cual deberá lograrse en los diferentes momentos de la misma. 
Como puede comprobarse estas tareas docentes hacen énfasis en la habilidad modelar, ya que el estudiante debe asociar a un objeto no matemático un objeto matemático, en este caso el límite de una función.

\section{Las ciencias biológicas y los modelos matemáticos}

La aplicación de herramientas matemáticas en el estudio de fenómenos, procesos y conceptos biológicos es una actividad de creciente importancia. A esta actividad se le llama Biología Matemática o Biomatemática. Su problema central es encontrar el método matemático más eficiente para solucionar o entender mejor un problema biológico. Es una ciencia multidisciplinaria que involucra a biólogos, matemáticos, físicos, químicos y fisiólogos, entre otros científicos. Hay dos niveles fundamentales en la relación biología-matemática: uno es el modelo matemático en biología y el otro, la biología teórica.

Un modelo matemático es una descripción matemática de un fenómeno del mundo real. Se encuentra determinado y definido por el problema que se quiere resolver, y por la pregunta que se quiere contestar (Miramontes, 1998). En el campo de las Ciencias Naturales los modelos matemáticos se han utilizado para describir el crecimiento de las poblaciones de animales, la concentración de un producto en una reacción química, el funcionamiento de las neuronas y la dinámica intracelular, por citar solo algunos ejemplos. La importancia del uso de modelos matemáticos para tratar de entender en profundidad el fenómeno y tal vez realizar alguna predicción sobre su comportamiento futuro, puede resumirse de la siguiente forma:

- Un biólogo puede pasarse toda la vida midiendo el tamaño y la distribución de una población sin lograr tener una idea de los mecanismos de interacción entre las variables medidas. Los modelos matemáticos ayudan a dar coherencia a la gran cantidad de datos experimentales o de campo que se obtienen del estudio de la naturaleza. Da una dirección y una idea sobre las variables más importantes que se miden, y proporciona un método para interpretar esos datos.

- Los modelos matemáticos ayudan a generar predicciones. Los biólogos harán progresos mucho más rápido en la comprensión de la naturaleza tratando de verificar o refutar predicciones específicas, que midiéndolo todo sin tener un objetivo para esas mediciones. Los modelos pueden también arrojar predicciones equivocadas.

Para ilustrar lo antes dicho tenemos las ecuaciones de Lotka-Volterra, también conocidas como ecuaciones predador-presa, son un par de ecuaciones diferenciales de primer orden no lineales que se usan para describir dinámicas de sistemas biológicos en el que dos especies 
interactúan, una como presa y otra como depredador. Las ecuaciones fueron propuestas de forma independiente por Alfred J. Lotka en 1925 y Vito Volterra en 1926. Tales ecuaciones se definen como:

$$
\begin{gathered}
\frac{d x}{d t}=x(\alpha-\beta y) \\
\frac{d y}{d t}=-y(\gamma-\delta x)
\end{gathered}
$$

Donde: $y$ es el número de algún predador (por ejemplo, un lobo); $x$ es el número de sus presas (por ejemplo, conejos); $d y / d t$ y $d x / d t$ representa el crecimiento de las dos poblaciones en el tiempo; $t$ representa el tiempo y $\alpha, \beta, \gamma$ y $\delta$ son parámetros que representan las interacciones de las dos especies. Estas ecuaciones permiten explicar ciertos fenómenos básicos de la dinámica poblacional de especies interactuantes.

- Los modelos matemáticos ayudan a los biólogos a distinguir los diferentes patrones que se ven en la naturaleza y a encontrar los mecanismos que causan ese comportamiento, ya que la matemática como ciencia se ocupa de identificar y clasificar patrones. Es por eso que los modelos matemáticos pueden esclarecer mecanismos biológicos.

El matemático Alan Turing se interesó en comprender la existencia de los números de Fibonacci en las estructuras vegetales. Para esto utilizó ecuaciones de reacción-difusión que actualmente son cruciales en el campo de la formación de patrones. En su trabajo "Fundamentos químicos de la morfogénesis" describe los mecanismos de formación de pautas espaciales de distribución de concentraciones de diversas sustancias o especies, como un conflicto entre la evolución espacialmente homogénea y la difusión de las especies llamadas ahora morfógenos, a través de las ecuaciones de reacción-difusión.

- Los modelos matemáticos permiten definir y probar una estrategia de solución para el control de poblaciones de animales, de los recursos naturales, de las enfermedades infecciosas, entre otros problemas. Por ejemplo, en nuestro país, se investiga en la búsqueda de modelos para predecir la dinámica de las poblaciones que afectan a cultivos de importancia económica, en virtud de establecer adecuadas estrategias para el manejo de plagas.

Por otra parte, resulta interesante saber que en el siglo XX el desarrollo de la teoría de ecuaciones diferenciales fue en gran medida estimulado, cuando no dirigido, por la necesidad de comprender fenómenos biológicos relevantes. Por ejemplo, el estudio de los mecanismos de 
transmisión de señales en el sistema nervioso influyó considerablemente en el desarrollo de la teoría de ondas viajeras en ecuaciones diferenciales (Hernandez, 2016).

Hoy en día, las ramas de estudio siguen creciendo, en Biología cada problema abordado es singular y, como resultado, las matemáticas que intervienen son muy variadas. En concreto, el uso de redes complejas en epidemiología requiere ideas de la teoría de grafos; el álgebra combinatoria se aplica en el control de sistemas en ecología; la rama topológica de la teoría de nudos describe la manera de anudarse de una molécula de $\mathrm{ADN}$; la investigación en genética molecular necesita de ciertos procesos estocásticos y los modelos lineales y no-lineales en neurobiología emplean ecuaciones diferenciales parciales deterministas y estocásticas.

Para el estudio de la Biología Matemática o Biomatemática se necesita un conocimiento básico de ecuaciones diferenciales, conocimiento que todo estudiante de la Licenciatura en Bioquímica y Biología Molecular obtiene a partir de las asignaturas Cálculo Diferencial y Cálculo Integral que se imparten en el primer año de la carrera. Con la intención de que los estudiantes de la carrera de Licenciatura en Bioquímica y Biología Molecular tuvieran claro desde el primer momento esa importante relación de la que acabamos de hablar, se les orientó desde el primer día de clases el siguiente Seminario.

\section{Seminario “¿Qué es la Biomatemática?”}

\section{Orientaciones al estudiante:}

Después de haber estudiado detenidamente el tema en cuestión, prepara una exposición de diez minutos que responda a las siguientes preguntas:

- ¿Qué es la Biomatemática?

- ¿ ¿Qué es un modelo matemático?

- ¿Cuál es su importancia?

- Ejemplifique cómo se utiliza la Biomatemática en el estudio del siguiente tema:

Los autores de este trabajo orientaron este seminario en el primer encuentro que tuvieron con sus estudiantes de primer año. Se orientó por equipos que fueron formados completamente al azar, en un momento en que todavía no estaban definidos los líderes de los grupos, lo cual influye de forma beneficiosa en las futuras relaciones interpersonales de los integrantes de los grupos. Esta característica se reflejó en la exposición oral, en el sentido en que fue grupal, todos 
los integrantes de cada equipo expusieron un fragmento de lo que había encontrado. A cada equipo se le dio un tema diferente para investigar. Los temas propuestos fueron:

- Estudio del crecimiento poblacional.

- Estudio del funcionamiento de las neuronas.

- Identificación y clasificación de patrones.

- La Ecología.

- Estudio del ADN.

- Estudio de la concentración de un producto en una reacción química.

- Estudio de la genética molecular.

- La transmisión de señales en el sistema nervioso.

- La estrategia para el manejo de plagas.

- Estudio de la dinámica intracelular.

El seminario tuvo un alto nivel científico. Los estudiantes se prepararon y mostraron iniciativas como presentar sus exposiciones utilizando las tecnologías de la información y las comunicaciones, en particular, presentaciones con PowerPoint. Después de realizado el seminario se les hizo una encuesta anónima para conocer sus opiniones.

Después de aplicada la encuesta, de los 45 estudiantes encuestados, 37 (82,2\%) opinaron que el Seminario los había motivado hacia la asignatura, $39(86,6 \%)$ lo encontraron interesante, y $32(71,1 \%)$ vinculado a su perfil. Finalmente el 93,3\% estuvo de acuerdo en que Tareas Docente como esta se debe incluir en el PEA de la asignatura, pues los motiva cada día más hacia el estudio de la misma.

\section{Experiencia comparativa para la sistematización de la optimización de funciones de variable real}

Innumerables son las oportunidades que brinda el PEA del Cálculo Diferencial para hacerle evidente al estudiante la vinculación de este contenido con su perfil. Para demostrar que, en efecto, los estudiantes se motivan más cuando realizan ejercicios con textos, donde las funciones modelan algún proceso vinculado con las Ciencias Naturales se diseñaron dos 
variantes para la Clase Práctica relacionada con la sistematización del cálculo de extremos de funciones de variable real.

\section{Variante I}

Calcule los extremos, si existen, de las siguientes funciones (Roldan, 2013):
a) $\quad f(x)=x+\sqrt{1-x}$
b) $f(x)=x e^{-x}$
c) $f(x)=3-2(x+1)^{\frac{1}{3}}$

\section{Variante II}

1. Hallar el radio de una tráquea contraída que logra que el aire salga a mayor velocidad cuando se tose utilizando el modelo matemático de la tos:

$$
v(r)=k\left(r_{0}-r\right) r^{2}
$$

$k$ - constante; $r_{0}$ - radio normal de la tráquea $\frac{1}{2} \leq r \leq r_{0}$

2. Se sabe que los peces intentan minimizar el gasto de energía por unidad de tiempo (E) cuando nadan contra la corriente a una velocidad v relativa al agua. La función

$$
E(v)=\frac{L a v^{2}}{v-u}
$$

Modela esa relación donde $u$ - constante, es la velocidad de la corriente; $L$ constante, es la distancia recorrida; $a$-constante de proporcionalidad $(a>o)$. Demuestre que existe un valor de v tal que el gasto de energía del pez (E) se hace mínima.

3. La rapidez en que la fotosíntesis tiene lugar para una especie de fitoplancton está modelada por la función:

$$
P(I)=\frac{100 I}{I^{2}+I+4}
$$

donde I es la intensidad de la luz. Calcule el valor de I para que P sea máxima. (Stewart, 2012)

Las dos variantes de Clase Práctica se aplicaron en dos subgrupos respectivamente. Antes y después de comenzar la clase se les hizo a los estudiantes una encuesta anónima, donde entre las preguntas a responder estaba la siguiente: En una escala del 1 al 5: Nada -1 Poco-2 Algo-3 Bastante-4 Mucho-5, escoja un número que defina su relación afectiva o motivación 
hacia la resolución de ejercicios de cálculo de extremos de una variable real. La tabla 1 que se presenta a continuación recoge el antes y el después de las respuestas de ambos grupos.

Tabla 1: Resultados de la encuesta

\begin{tabular}{lccccc}
\hline & Nada & Poco & Algo & Bastante & Mucho \\
\hline Grupo Control- Antes & 0 & 3 & 12 & 3 & 1 \\
Grupo Control - Después & 1 & 0 & 9 & 8 & 1 \\
Otro-Grupo Antes & 1 & 5 & 7 & 3 & 1 \\
Otro Grupo - Después & 0 & 0 & 7 & 7 & 3 \\
\hline
\end{tabular}

En el Gráfico 1 se compara el antes y el después de la aplicación de la Variante I en el Grupo Control:

Gráfico 1: Resultados de la aplicación de la Variante I
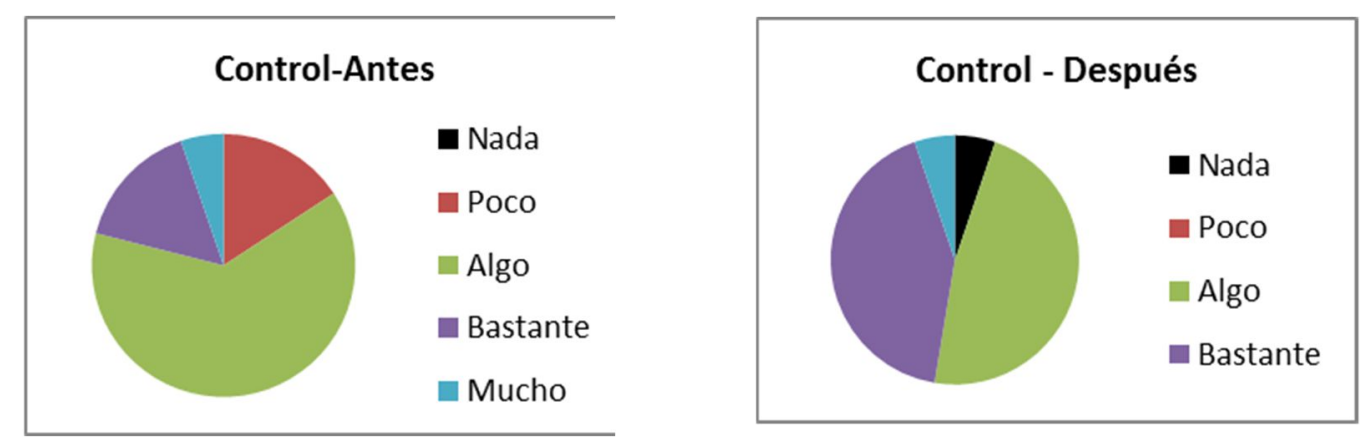

Gráfico 2: Resultados de la aplicación de la Variante II
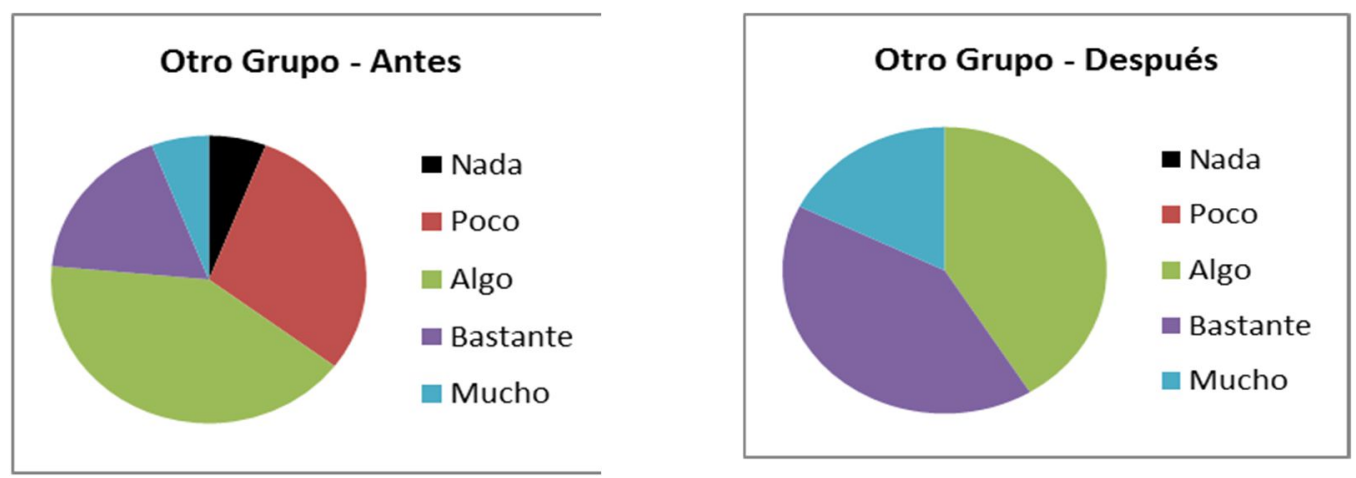

Se observa que en el subgrupo donde se aplicó la variante II, es decir, los ejercicios contextualizados a problemas biológicos, se alcanzaron mejores logros en cuanto a la motivación hacia el contenido impartido. Los resultados de este experimento confirman una vez más que si los problemas o tareas que se propongan al estudiante están vinculados con su perfil vocacional, se está contribuyendo a que el aprendizaje llegue a ser significativo y desarrollador, objetivo que debe regir cualquier PEA. 


\section{Conclusiones}

El profesor que imparte Matemática en el contexto de las Ciencias Biológicas debe conocer la relación entre la Matemática y la Biología para crear vínculos afectivos con los estudiantes a través del uso de un lenguaje común y para poder potenciar el interés de los mismos hacia su asignatura.

Es responsabilidad del profesor convertir la Tarea Docente en una herramienta fundamental para lograr un PEA eficaz y desarrollador. Para esto debe diseñarla de forma tal que en su ejecución, se establezca una relación afectiva entre el objetivo de la tarea y los intereses del estudiante, creando en el aula escenarios activos de conocimiento compartido y que éste se sienta parte activa de su propio aprendizaje.

Si nos limitamos a que nuestros estudiantes aprendan a derivar, sin hacer énfasis en los ejercicios de aplicación de esta potente herramienta, la función desarrolladora queda trunca, y no se aprovecha este conocimiento para crear un nexo entre lo estudiado y el perfil de profesional. Se habrá cumplido con el incremento de las capacidades cognoscitivas del estudiante. Convencerlo de que a través del Cálculo Diferencial puede ser creativo y transformar la sociedad en la que vive, solo será posible a través de problemas de aplicación.

\section{Agradecimientos o reconocimientos}

Trabajo elaborado en el marco del proyecto de investigación: Aprendizaje desarrollador desde el proceso de enseñanza aprendizaje de la Matemática, que responde al Programa Nacional de Ciencias Básicas, del Ministerio de Educación Superior en Cuba y al Grupo de Enseñanza de la Matemática de la Universidad de la Habana, formado por docentes de matemática del departamento de Matemática de Universidad de la Habana.

\section{Referencias}

Hernandez, M. (2016). Matemáticas aplicadas a la Biologia. Revista Iberoamericana de Educacion Matematica: Union. No 45. 24-43

Miramontes, P. (1998). La Biologia Matematica . Grupo de Biomatematica. Facultad de Ciencias. UNAM. Mexico.

Roldan, R. (2013). Matemática I para Ciencias Naturales. La Habana: Editorial Felix Varela.

Stewart, J. (2012). Calculo de una variable. Trascendentes tempranas. Cengage Learning Editores. 
Autores:

Reinaldo Sampedro Ruiz es Profesor Titular y forma parte del claustro de docentes de la Facultad de Matematica Computación MATCOM, de la Universidad de la Habana. Doctor en Ciencias Pedagógicas y desarrolla investigaciones en las líneas de didáctica de la matemática, desarrollo profesional docente y competencias relacionadas con la gestión del conocimiento matemático. Ha colaborado en la superación del claustro profesoral en varias universidades de la República de Venezuela, la República Dominicana y la República Popular de Angola. Es autor de varios artículos relacionados con la formación matemática de los estudiantes de carreras de ingeniería y ciencias médicas.

María Elvira Fernández $\boldsymbol{S a}$ es Profesora Asistente y forma parte del claustro de docentes de la Facultad de Matemática Computación MATCOM, de la Universidad de la Habana. Master en Didáctica de la Matemática y desarrolla investigaciones en las líneas de didáctica de la matemática y desarrollo profesional docente. Ha colaborado en la superación del claustro profesoral en varias universidades de Cuba. Es autora de varios artículos relacionados con la formación matemática de los estudiantes de carreras de Ciencias Biológicas.

Marleny Soler Martínez es Profesora Titular y forma parte del claustro de docentes de la Facultad de Matemática Computación MATCOM, de la Universidad de la Habana. Doctor en Ciencias Pedagógicas y desarrolla investigaciones en las líneas de didáctica de la matemática, desarrollo profesional docente y competencias relacionadas con la gestión del conocimiento matemático. Ha colaborado en la superación del claustro profesoral en varias universidades de la República Popular de Angola. Es autora de varios artículos relacionados con la formación matemática de los estudiantes de carreras de Ciencias Biológicas. 